\title{
On Locally Gabriel Geometric Graphs
}

\author{
Sathish Govindarajan * Abhijeet Khopkar $^{\dagger}$
}

\begin{abstract}
Let $P$ be a set of $n$ points in the plane. A geometric graph $G$ on $P$ is said to be locally Gabriel if for every edge $(u, v)$ in $G$, the disk with $u$ and $v$ as diameter does not contain any points of $P$ that are neighbors of $u$ or $v$ in $G$. A locally Gabriel graph is a generalization of Gabriel graph and is motivated by applications in wireless networks. Unlike a Gabriel graph, there is no unique locally Gabriel graph on a given point set since no edge in a locally Gabriel graph is necessarily included or excluded. Thus the edge set of the graph can be customized to optimize certain network parameters depending on the application. In this paper, we show the following combinatorial bounds on edge complexity and independent sets of locally Gabriel graphs:
\end{abstract}

(i) For any $n$, there exists locally Gabriel graphs with $\Omega\left(n^{5 / 4}\right)$ edges. This improves upon the previous best bound of $\Omega\left(n^{1+\frac{1}{\log \log n}}\right)$.

(ii) For various subclasses of convex point sets, we show tight linear bounds on the maximum edge complexity of locally Gabriel graphs.

(iii) For any locally Gabriel graph on any $n$ point set, there exists an independent set of size $\Omega(\sqrt{n} \log n)$.

\section{Introduction}

A geometric graph $G=(V, E)$ is an embedding of the set $V$ as points in the plane and edges in $E$ as straight-line segments connecting the points in $V$. Delaunay graphs, Gabriel graphs and Relative Neighborhood graphs (RNG) are fundamental geometric proximity graphs with applications in fields like computer graphics, vision, GIS, wireless networks, etc. For a nice survey on these graphs and their applications, see [11].

The Gabriel graph introduced by Gabriel and Sokal [9] is defined as follows: Given a set of points $P$ in the plane, an edge exists between points $u$ and $v$ iff the Euclidean disk with $u$ and $v$ as diameter does not contain any other point of $P$. Gabriel graphs have been used to model the topology in wireless networks [3, 18]. Motivated by applications in wireless networks, [14, 12] generalized these structures to $k$-locally delaunay/gabriel graphs. The edge complexity of these structures have been studied in

\footnotetext{
${ }^{*}$ Department of Computer Science and Automation, Indian Institute of Science, Bangalore, India. E-mail: gsat@csa.iisc.ernet.in

${ }^{\dagger}$ Department of Computer Science and Automation, Indian Institute of Science, Bangalore, India. E-mail: abhijitecsa.iisc.ernet.in
} 
[12, 15]. In this paper, we focus on 1-locally Gabriel graphs and call them as Locally Gabriel Graphs (LGGs).

A locally gabriel graph is a geometric graph $G$ with the following property: for each edge $(u, v)$ in $G$, the Euclidean disk with $u$ and $v$ as diameter does not contain any points of $P$ that are neighbors of $u$ or $v$ in $G$.

Study of these graphs was initially motivated by design of dynamic routing protocols for ad hoc wireless networks [13]. An ad-hoc wireless network consists of a collection of wireless transceivers (corresponds to points) and an underlying network topology (corresponds to edges) that is used for communication/routing. Like Gabriel Graphs, $L G G s$ can be used to design wireless network topology since they capture the interference patterns well. An interesting point to be noted is that there is no unique $L G G$ on a given point set since no edge in $L G G$ is necessarily included or excluded. Thus the edge set of the graph (used for wireless communication) can be customized to optimize certain network parameters depending on the application. LGGs also provide certain advantages over Gabriel Graphs. While a Gabriel graph has linear number of edges (planar graph), we show in this paper that there exists $L G G$ s with $n^{5 / 4}$ edges. A dense network can be desirable for applications like broadcasting or multicasting where a large number of pairs of nodes need to communicate with each other. Another important parameter in the topology of wireless network is the number of simultaneous transmissions that can be performed. A node in a wireless network cannot transmit and receive in the same time slot. Thus, the set of transmitting nodes at any time slot form an independent set in the underlying graph. We show that there exists an independent set of size $\Omega(\sqrt{n} \log n)$ in any $L G G$ of any $n$ pointset.

An interesting combinatorial question, that we address in this paper, is to bound the edge complexity of locally gabriel graphs.

It was observed in [15] that the unit distance graph [7], introduced by Erdos, is also a locally delaunay/gabriel graph. The maximum edge complexity of unit distance graphs has been extensively studied [7, 16, 17]. See [4] for a survey on this problem. There is a significant gap between the lower and upper bounds and improving them is considered a hard open problem in discrete geometry. The edge complexity of unit distance graphs on convex point sets have also been studied. The best lower bound is $2 n-7$ [6] and the best upper bound is $n \log n$ [8, 5]. It has been conjectured in [4] that the edge complexity of unit distance graphs on convex point sets is $2 n$.

[12] initiated the study of maximum edge complexity of locally delaunay/gabriel graphs by showing non-trivial upper bounds. [15] showed an upper bound of $O\left(n^{3 / 2}\right)$ and a lower bound of $\Omega\left(n^{4 / 3}\right)$ on the maximum edge complexity of locally delaunay graphs.

For locally gabriel graphs, [12] showed an upper bound of $O\left(n^{3 / 2}\right)$ by proving that $K_{2,3}$ is a forbidden subgraph. The best known lower bound is $\Omega\left(n^{1+\frac{1}{\log \log n}}\right)$ [7], given by Erdos classic lower bound construction for unit distance graphs. While the gap between the upper and lower bounds for locally delaunay graphs has been narrowed significantly, the gap is quite wide for locally gabriel graphs. In this paper, we improve the lower bound significantly.

We show the following results in this paper:

(i) For any $n$, there exists locally gabriel graphs with $\Omega\left(n^{5 / 4}\right)$ edges. This improves 
the previous lower bound of $\Omega\left(n^{1+\frac{1}{\log \log n}}\right)$ [7].

(ii) For various subclasses of convex point sets like monotonic convex point set, half convex point set, centrally symmetric convex point set, we prove tight linear bounds on the edge complexity of locally gabriel graphs.

(iii) For any $L G G$ on any $n$ point set, we show that there exists an independent set of size $\Omega(\sqrt{n} \log n)$.

The paper is organized as follows: Definitions that will be used in the paper is presented in Section 2. We present the lower bound construction in Section 3 and analyze it in Section 4. We prove various upper and lower bounds for convex point sets in Section 5. The independent set construction is presented in Section 6.

\section{Preliminaries}

Let $P$ be a set of $n$ points in $\mathbb{R}^{2}$. For any $p, q \in P$, we denote by $d_{p q}$ the disk with $p$ and $q$ as diameter.

Definition 2.1 (Locally Gabriel condition) Let $G_{P}$ be a geometric graph on $P$. An edge $(u, v)$ of $G_{P}$ is said to satisfy the locally Gabriel condition if disk $d_{u v}$ does not contain neighbors of $u$ or $v$ in $G_{P}$.

Definition 2.2 (Locally Gabriel Graph) A geometric graph $G_{P}$ on P is said to be Locally Gabriel Graph (LGG) if every edge of $G_{P}$ satisfies the locally Gabriel condition.

Let $p=\left(p^{x}, p^{y}\right)$ be any point in $\mathbb{R}^{2}$.

Definition 2.3 (Upper-right monotonic convex point set) Let $P=\left\{p_{1}, p_{2} \ldots, p_{k}\right\}$ be a set of points in convex position that are ordered in counterclockwise direction. $P$ is called a upper-right monotonic convex point set if $p_{i}^{x} \leq p_{j}^{x}, p_{i}^{y} \geq p_{j}^{y}, \forall 1 \leq i<j \leq k$

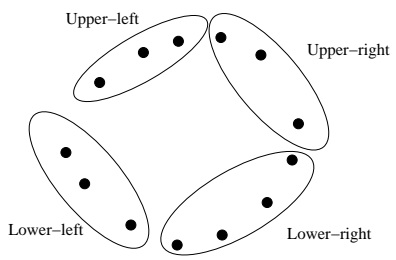

Figure 1: Four types of monotonic convex sets

Similarly, we define the other three types of monotonic convex point sets, i.e., upper-left, lower-right and lower-left. Figure 1 shows the 4 types of monotonic convex point sets. Note that any convex point set can be decomposed into the above 4 types.

Definition 2.4 (Half convex point set) Let $P=Q \cup R$ be a set of points in convex position that is ordered in counterclockwise direction. $P$ is called a right (resp. left) half convex point set if $Q$ is upper-right monotonic and $R$ is lower-right monotonic (resp. $Q$ is upper-left monotonic and $R$ is lower-left monotonic). 
Definition 2.5 (Centrally symmetric convex point set) Let $P$ be a set of points in convex position. $P$ is said to be centrally symmetric with respect to the origin, if for every point $p \in P$, point $-p$ also belongs to $P$

Let $p, q, r$ be three points in $P$.

Lemma 2.1 If $q$ and $r$ are neighbors of $p$ in an $L G G$ on $P$, then $\angle p q r, \angle p r q<\pi / 2$.

Proof. Since $(p, q)$ is an edge of $G_{P}, r$ must lie outside the disk $d_{p q}$. Thus, $\angle p r q<\frac{\pi}{2}$. Since $(p, r)$ is also an edge in $G_{P}, q$ must lie outside the disk $d_{p r}$. Thus, $\angle p q r<\frac{\pi}{2}$.

Conversely, if either $\angle p q r \geq \frac{\pi}{2}$ or $\angle p r q \geq \frac{\pi}{2}$, then we call the edges $(p, q)$ and $(p, r)$ as conflicting. Two conflicting edges cannot exist simultaneously in an LGG.

\section{Lower Bound Construction}

In this section, we describe the construction of a $L G G$ with $\Omega\left(n^{5 / 4}\right)$ edges. The point set $P$ for this construction is a $\sqrt{n} \times \sqrt{n}$ uniform grid. First, we describe the algorithm that constructs the $L G G G_{P}$ on the grid point set $P$. Then, we prove the correctness of our algorithm. Finally, we analyze the edge complexity of $G_{P}$.

\subsection{Construction}

Let us denote the points on the grid as $(x, y), 0 \leq x, y<\sqrt{n}$. The algorithm is an iterative greedy procedure that assigns neighbors to each grid point. First, we describe the procedure that assigns neighbors to an arbitrary point $p=\left(p^{x}, p^{y}\right)$ on the grid. For technical reasons, we only assign neighbors to $p$ that are in the first and third quadrant w.r.t. $p$. By applying this procedure to the grid points $(x, y), \sqrt{n} / 3 \leq x, y<2 \sqrt{n} / 3$ (we choose only these grid points to avoid edge effects), we obtain our $L G G G_{P}$.

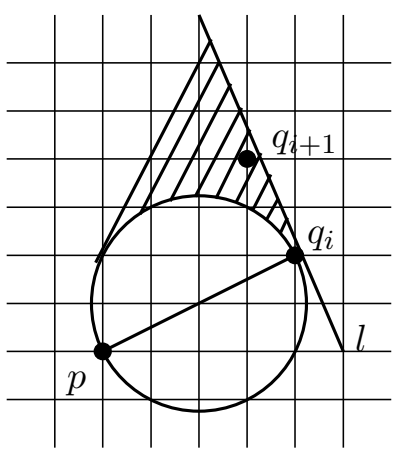

Figure 2: Feasibility region for the next neighbor $q_{i+1}$

Now, we describe the iterative procedure that assigns neighbors to $p$ in a counterclockwise direction. Let $q_{i}$ be the current neighbor of $p$ that is assigned by the procedure and $\theta_{i}$ be the angle that segment $p q_{i}$ makes with the positive direction of $\mathrm{x}$-axis. 
First, we describe how to find the next neighbor $q_{i+1}$ in the counter-clockwise direction. Let us describe the feasibility region for $q_{i+1}$. Figure 2 shows the points $p, q_{i}$, the disk $d_{p q_{i}}$ and the tangent line $l$ at $q_{i}$. Since $\left(p, q_{i}\right)$ is an edge in $G_{P}, q_{i+1}$ must lie outside $d_{p q_{i}}$. Also, since $\left(p, q_{i+1}\right)$ will be an edge in $G_{P}, \angle p q_{i} q_{i+1}<\frac{\pi}{2}$ (by Lemma2.1). This implies that $q_{i+1}$ must lie below the tangent line $l$. Thus the feasible region for $q_{i+1}$ is outside $d_{p q_{i}}$ and below $l$ (shown as the shaded region in Figure 2). We choose the next neighbor $q_{i+1}$ to be the grid point in the feasible region that is closest (in Euclidean distance) to $q_{i}$ (See Figure 2]). This greedy choice allows us to pack as many neighbors as possible.

Now, the procedure that assigns neighbors to $p$ is as follows: The first neighbor of $p$ is set as $q_{0}=\left(p^{x}+s, p^{y}+s \cdot \tan \theta_{0}\right)$, where $s=\sqrt{n} / 3$ and $\theta_{0}, 0<\theta_{0}<\pi / 4$ is a small constant to be fixed later. Starting with this neighbor, we iteratively find the next neighbor using the procedure described above. We continue assigning neighbors as long as the condition $\theta_{i} \leq \pi / 4$ is satisfied. Note that this procedure assigns neighbors only in the first quadrant w.r.t $p$. Similarly, we find neighbors in the third quadrant w.r.t $p$ by starting with the initial neighbor $\left(p^{x}-s, p^{y}-s \cdot \tan \theta_{0}\right)$ and proceeding as long as the condition $\theta_{i} \leq 5 \pi / 4$ is satisfied.

\subsection{Correctness}

In this section, we show that the geometric graph $G_{P}$ constructed above is a locally gabriel graph.

Remark 1: Observe that the above procedure that constructs $G_{P}$ assigns neighbors in a symmetric consistent manner, i.e., if the procedure assigns $q_{i}$ as the $i$-th neighbor (in 1st quadrant) of $p$, then it would assign $p$ as the $i$ th neighbor (in 3rd quadrant) of $q_{i}$, when the procedure is applied on $q_{i}$.

By Remark 1, the neighbors of $p$ in $G_{P}$ are exactly the grid points chosen by the procedure.

Lemma 3.1 Let $p \in P$ be any grid point and let $Q=\left\{q_{0}, q_{1}, \ldots, q_{m}\right\}$ be the neighbors of $p$ in $G_{P}$ (in counter-clockwise order) in the first quadrant. The disk $d_{p q_{i}}$ does not contain any neighbor of $p \forall i, 0 \leq i \leq m$.

Proof. First, we show that $d_{p q_{i}}$ does not contain any neighbor of $p$ in the first quadrant, i.e., $d_{p q_{i}} \cap\left(Q \backslash\left\{q_{i}\right\}\right)=\emptyset$. Observe that $d_{p q_{i}}$ does not contain $q_{i+1}$ because the iterative procedure picks $q_{i+1}$ outside the disk $d_{p q_{i}}$. Also observe that $d_{p q_{i}}$ does not contain $q_{i-1}$ because $\angle p q_{i-1} q_{i}<\frac{\pi}{2}$ ( $q_{i}$ is picked below tangent line of $d_{p q_{i-1}}$ ). On the contrary, let us assume that $d_{p q_{i}}$ contains some $q_{j}, j \neq i-1, i, i+1$. There are 2 cases: (i) $j>i+1$ and (ii) $j<i-1$. We will prove case (i) below. Case (ii) can be proved in a similar manner. Let us assume that $k$ is the smallest index among the neighbors $q_{j}, j>i+1$ that is contained in $d_{p q_{i}}$. Since $q_{i}, q_{i+1}, \ldots q_{k-1}, q_{k}$ are in counter-clockwise convex position, all the disks $d_{p q_{j}}, i+1 \leq j \leq k-1$ also contains $q_{k}$ (see figure 3). Thus, the disk $d_{p q_{k-1}}$ also contains $q_{k}$. This is a contradiction since the iterative procedure picks $q_{k}$ outside the disk $d_{p q_{k-1}}$.

The disk $d_{p q_{i}}$ does not contain any neighbor of $p$ in the third quadrant w.r.t $p$, since $d_{p q_{i}}$ does not intersect the third quadrant w.r.t $p$. Thus $d_{p q_{i}}$ does not contain any neighbor of $p$. 


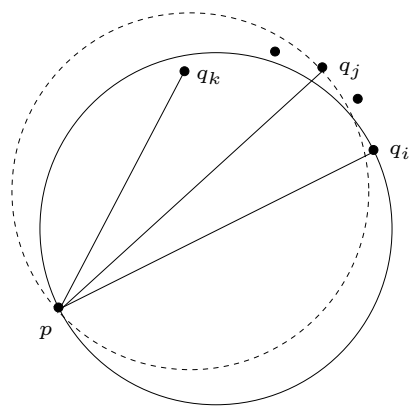

Figure 3: Point $p$ and its neighbors $q_{i}, \ldots, q_{j}, \ldots, q_{k}$

Remark 2: Observe that the grid point set $P$ is a symmetric point set and we use the same deterministic procedure to assign neighbors to all the grid point. Hence Lemma 3.1 is true for all the grid points $p \in P$.

Lemma 3.2 Edge $\left(p, q_{i}\right)$ of $G_{P}$ satisfies the locally Gabriel condition $\forall i, 0 \leq i \leq m$.

Proof. We need to show that the disk $d_{p q_{i}}, 0 \leq i \leq m$, does not contain the neighbors of $p$ or $q_{i}$ in $G_{P}$. By Lemma 3.1, disk $d_{p q_{i}}$ does not contain any neighbor of $p$.

By Remark $1, p$ is the $i$ th neighbor (in 3rd quadrant) of $q_{i}$. By Remark 2, we apply Lemma 3.1 for grid point $q_{i}$ (instead of $p$ ) on the neighbors of $q_{i}$ in the 3rd quadrant (instead of 1st quadrant) to show that disk $d_{q_{i} p}$ (which is the same as $d_{p q_{i}}$ ) does not contain any neighbors of $q_{i}$.

Since the procedure assigns neighbors to $p$ in the third quadrant in exactly the same way as the first quadrant, Lemma 3.2 shows that edges from $p$ to its neighbors in the third quadrant also satisfy the locally Gabriel condition. Thus, all the edges from $p$ to neighbors of $p$ satisfies the locally Gabriel condition. Since we use the same deterministic procedure to assign neighbors to all the grid points, the argument for $p$ applies to all grid points $p \in P$. Hence all the edges in $G_{P}$ satisfy the locally Gabriel condition proving that $G_{P}$ is locally Gabriel.

\subsection{Analysis}

In this section, we analyze the lower bound construction described in the previous section. We will show that $G_{P}$ has $\Omega\left(n^{5 / 4}\right)$ edges by proving that the iterative procedure picks $\Omega\left(n^{1 / 4}\right)$ neighbors for grid point $p$. Let $q_{0}, q_{1}, \ldots, q_{m}$ be the neighbors(in counter-clockwise order) of $p$ in the first quadrant. Given the current neighbor $q_{i}$, the procedure picks the next neighbor $q_{i+1}$ "close" to $q$. We will prove bounds on the closeness between $q_{i}$ and $q_{i+1}$. Using this, we show bounds on $m$.

Figure 4 shows the points $p$ (denoted as $\mathrm{A}$ in the figure), the current neighbor $q_{i}$ (denoted as $\mathrm{C}$ ), the disk $d_{p q_{i}}$ and the tangent line $l$ at $\mathrm{C}$. Let the next neighbor $q_{i+1}$ lie at a x-distance $d_{i}$ from the current neighbor $q_{i}\left(q_{i+1}\right.$ lies on the vertical line passing through D and E). Let $|A B|=q_{i}^{x}-p^{x}=x_{i},|D B|=q_{i}^{x}-q_{i+1}^{x}=d_{i}$ and $|C B|=$ 


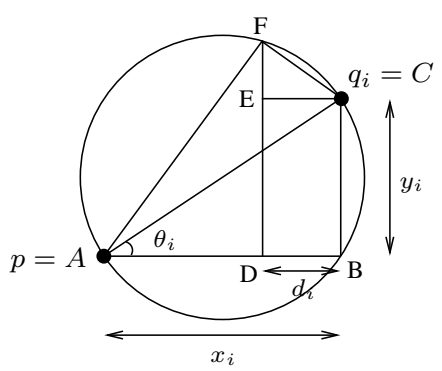

(i)

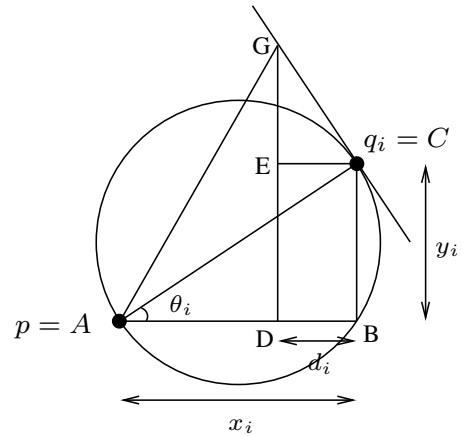

(ii)

Figure 4: The vertical gridline that contains the next neighbor intersects (i) the diameter disk at $F$, (ii) the tangent line at $G$

$q_{i}^{y}-p^{y}=y_{i}$ (See Figure 44). First, we will prove bounds for $d_{i}$ in terms of $x_{i}$. Let the vertical grid-line passing through $q_{i+1}$ intersect the disk $d_{p q_{i}}$ at $F$ (See Figure 4 (i)) and the tangent line at $G$ (See Figure 4 (ii)). Let $|F E|=h_{i}$ and $|G E|=h_{i}^{\prime}$. Since $\triangle A F C$ is right-angled at $F$ (see Figure 4(i)), we have

$$
\begin{aligned}
|A C|^{2} & =|A F|^{2}+|F C|^{2} \\
& =\left(|A D|^{2}+|D F|^{2}\right)+\left(|F E|^{2}+|C E|^{2}\right) \\
\left(x_{i} \sec \theta_{i}\right)^{2} & =\left(x_{i}-d_{i}\right)^{2}+\left(h_{i}+x_{i} \tan \theta_{i}\right)^{2}+h_{i}^{2}+d_{i}^{2}
\end{aligned}
$$

Simplifying, we get

$$
h_{i}^{2}+x_{i} \tan \theta_{i} \cdot h_{i}-d_{i}\left(x_{i}-d_{i}\right)=0
$$

Similarly, since $\triangle A C G$ is right-angled at $C$ (see Figure 4(ii)), we have

$$
\begin{aligned}
|A G|^{2} & =|A C|^{2}+|C G|^{2} \\
\left(|A D|^{2}+|D G|^{2}\right) & =|A C|^{2}+\left(|G E|^{2}+|C E|^{2}\right) \\
\left(x_{i}-d_{i}\right)^{2}+\left(h_{i}^{\prime}+x_{i} \tan \theta_{i}\right)^{2} & =\left(x_{i} \sec \theta_{i}\right)^{2}+{h_{i}^{\prime}}^{2}+d_{i}^{2}
\end{aligned}
$$

Simplifying, we get $h_{i}^{\prime}=d_{i} \cot \theta_{i}$.

The next neighbor $q_{i+1}$ lies on the vertical gridline between $F$ and $G$. To ensure that a grid point exists between $F$ and $G$, we enforce a stronger condition that the distance between $F$ and $G$ is at least 1, i.e., $|F G|=h_{i}^{\prime}-h_{i}>1$. Solving for $h_{i}$ in Equation11 substituting for $h_{i}, h_{i}^{\prime}$, we get

$$
d_{i} \cot \theta_{i}-\frac{\sqrt{x_{i}^{2} \tan ^{2} \theta_{i}+4 d_{i}\left(x_{i}-d_{i}\right)}-x_{i} \tan \theta_{i}}{2}>1
$$

Simplifying this, we get the inequality

$$
d_{i}^{2}+\sin ^{2} \theta_{i}>x_{i} \tan \theta_{i} \sin ^{2} \theta_{i}+d_{i} \sin 2 \theta_{i}
$$


By setting $d_{i}=c_{1} \sqrt{x_{i}}, c_{1}>1$, the above inequality is satisfied, since $\theta_{i} \leq \pi / 4$ (we assign neighbors to $p$ only till $\theta_{i} \leq \pi / 4$ ). Therefore, inequality 2 is also satisfied. This gives us a bound on $d_{i}$ (closeness between $q_{i+1}$ and $q_{i}$ ) in terms of $x_{i}$ (x-distance of $q_{i}$ from $p$ ).

Now, we will obtain bounds on $m$, the number of neighbors assigned to $p$. Note that the procedure assigns neighbors to $p$ as long as $\theta_{i} \leq \pi / 4$, i.e., $y_{m} \leq x_{m}$. We will now obtain bounds on $x_{i}$ and $y_{i}$. The $x_{i}$ are related by the following recurrence relation

$$
\begin{aligned}
x_{i+1} & =x_{i}-d_{i} \\
& =x_{i}-c_{1} \sqrt{x_{i}} \\
& \geq x_{i}-c_{1} \sqrt{\frac{\sqrt{n}}{3}} \quad\left(x_{i} \leq \frac{\sqrt{n}}{3}\right)
\end{aligned}
$$

Expanding this recurrence with $x_{0}=\sqrt{n} / 3$, we get

$$
x_{k} \geq \frac{\sqrt{n}}{3}-\frac{k \cdot c_{1} n^{1 / 4}}{\sqrt{3}}, 0<k \leq m
$$

Next, we obtain bounds on $y_{i}$. The $y_{i}$ are related by the recurrence relation $y_{i+1}=y_{i}+$ $\left\lfloor h_{i}+1\right\rfloor$ (since we pick $q_{i+1}$ as the closest grid point to F). Expanding this recurrence, we get

$$
\begin{aligned}
y_{k} & =y_{0}+\sum_{0}^{k-1}\left\lfloor h_{i}+1\right\rfloor \\
& \leq y_{0}+k+\sum_{0}^{k-1} h_{i}
\end{aligned}
$$

where $h_{i}$ is given by the solution to Equation 1

$$
\begin{aligned}
\sum_{0}^{k-1} h_{i} & =\frac{1}{2} \sum_{0}^{k-1} \sqrt{x_{i}^{2} \tan ^{2} \theta_{i}+4 d_{i}\left(x_{i}-d_{i}\right)}-x_{i} \tan \theta_{i} \\
& =\frac{1}{2} \sum_{0}^{k-1} \sqrt{x_{i}^{2} \tan ^{2} \theta_{i}+4 c_{1} \sqrt{x_{i}}\left(x_{i}-c_{1} \sqrt{x_{i}}\right)}-x_{i} \tan \theta_{i} \\
& =\frac{1}{2} \sum_{0}^{k-1} x_{i} \tan \theta_{i}\left(\sqrt{1+\frac{4 c_{1} \sqrt{x_{i}}\left(x_{i}-c_{1} \sqrt{x_{i}}\right)}{x_{i}^{2} \tan ^{2} \theta_{i}}}-1\right) \\
& \leq \frac{1}{2} \sum_{0}^{k-1} x_{i} \tan \theta_{i}\left(\sqrt{1+\frac{4 c_{1}}{\sqrt{x_{i}} \tan ^{2} \theta_{i}}}-1\right) \\
& \leq \frac{1}{2} \sum_{0}^{k-1} x_{i} \tan \theta_{i}\left(\left(1+\frac{2 c_{1}}{\sqrt{x_{i}} \tan ^{2} \theta_{i}}\right)-1\right) \\
& \leq \sum_{0}^{k-1} \frac{c_{1} \sqrt{x_{i}}}{\tan \theta_{i}}
\end{aligned}
$$


Since $\theta_{i}>\theta_{0}$ and $x_{i} \leq \sqrt{n} / 3$, we have

$$
\sum_{0}^{k-1} h_{i} \leq \frac{c_{1} \cdot k \cdot n^{1 / 4}}{\sqrt{3} \tan \theta_{0}}
$$

Hence, from Equation 5, $y_{k}$ is given by the following

$$
y_{k} \leq \frac{\tan \theta_{0} \cdot \sqrt{n}}{3}+\frac{c_{1} \cdot k \cdot n^{1 / 4}}{\sqrt{3} \tan \theta_{0}}+k
$$

Setting $c_{1}=1.01, \theta_{0}=1.74 \times 10^{-3}$, it can be verified analytically in Equation 3 and Equation 6 that $y_{k} \leq x_{k}$ for all $0 \leq k \leq 10^{-4} n^{1 / 4}$. Thus, $y_{m} \leq x_{m}$ for $m=$ $10^{-4} n^{1 / 4}$. The number of neighbors of $p$ is at least $10^{-4} n^{1 / 4}$. The edge complexity of $G_{P}$ is therefore $\Omega\left(n \cdot n^{1 / 4}\right)=\Omega\left(n^{5 / 4}\right)$.

\section{Convex Point Sets}

In this section, we show edge complexity for LGG on various classes of convex point sets. First, we show exact bounds for half convex point sets. Then, we show asymptotic tight linear bounds for special subclasses of convex point sets. Finally, we show $O(n \log n)$ bounds for arbitrary convex point sets.

\subsection{Exact Bound for Half Convex Point Sets}

First, let us consider the special case when $P$ is a monotonic convex point set. Wlog, let us assume that $P$ is of the upper-right type.

Lemma 4.1 Let $P=\left\{p_{1}, p_{2}, \ldots, p_{n}\right\}$ be a upper-right monotonic convex point set and let $G_{P}$ be any locally gabriel graph on $P . p_{1}, p_{n}$ has atmost 1 neighbor in $G_{P}$ and hence $G_{P}$ has atmost $n-1$ edges.

Proof. We show that the first point $p_{1}$ has atmost one neighbor. Let if possible, $p_{i}$ and $p_{j}$ be neighbors of $p_{1}, j>i . p_{1}, p_{i}, p_{j}$ are in monotonic convex position. Thus $\angle p_{1} p_{i} p_{j} \geq 90^{\circ}$. Since $p_{i}$ and $p_{j}$ are neighbors of $p_{1}, \angle p_{1} p_{i} p_{j}<90^{\circ}$ (by Lemma2.1). Hence a contradiction. By a similar argument, we can also show that $p_{n}$ has atmost 1 neighbor in $G_{P}$.

Removing $p_{1}$ from $P$ and applying induction on the remaining points, we see that $G_{P}$ has atmost $n-1$ edges.

Next we consider the special case when $P$ is a half convex point set. Wlog, let us assume that $P$ is a right half convex point set.

Lemma 4.2 Let $P=Q \cup R$ be a right half convex point set with $n$ points, where $Q$ is upper-right monotonic and $R$ is lower-right monotonic. Let $G_{P}$ be any locally gabriel graph on $P$. $G_{P}$ has atmost $2 n-3$ edges. 
Proof. Let $p$ be the point with maximum x-coordinate (rightmost point) in $P . Q \cup\{p\}$ is upper-right monotonic and $R \cup\{p\}$ is lower-right monotonic. By Lemma 4.1 $p$ has degree atmost two(atmost one neighbor in $Q$ and one in $R$ ). Removing $p$ from $P$ and applying induction on the remaining points, we get $P(n) \leq P(n-1)+2 ; P(2)=1$. This gives $P(n) \leq 2 n-3$.

The above bounds are tight, i.e., it is easy to construct locally gabriel graphs for monotonic and half convex sets that match the above bounds. For any monotonic convex sets, construct a path (of length $n-1$ ) connecting all the vertices.

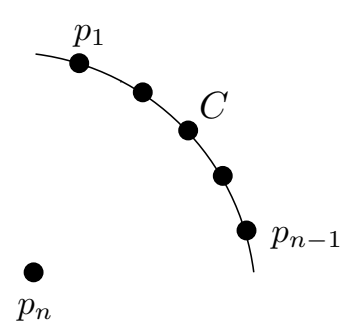

Figure 5: Portion of circle $\mathrm{C}$ centered at $p_{n}$ and points $p_{1}, \ldots, p_{n-1}$ placed equidistant on $C$

For right half convex sets, we can achieve the exact bound using the following construction:

Let $C$ be a circle with center at $p_{n}$. We place points $p_{1}, p_{2}, \ldots, p_{n-1}$ equidistant along the first quadrant of $C$ (See Figure 5). The point set constructed is right half convex. The edges of $G_{P}$ are defined as follows:

(i) Add edges $\left(p_{i}, p_{i+1}\right), 1 \leq i \leq n-2$. This forms a path of length $n-2$.

(ii) Add edges $\left(p_{n}, p_{i}\right), 1 \leq i \leq n-1$. This forms a star of size $n-1$.

It can be verified that these edges satisfy the locally gabriel condition. Thus, the edge complexity of $G_{P}$ is $2 n-3$.

\subsection{Tight Linear Bounds for Various Subclasses}

In this section, we prove asymptotic tight linear bounds for some special subclasses of convex point sets.

\subsubsection{Points on a Circle}

First, we consider the special case of $n$ points lying on a circle.

Lemma 4.3 Let $C$ be any circle and $P=\left\{p_{1}, p_{2}, \ldots, p_{n}\right\}$ be $n$ points that lie on $C$. Let $G_{P}$ be any locally gabriel graph on $P . G_{P}$ has atmost $n$ edges 
Proof. Let $p_{i}$ be any point in $P$ and $p_{i}^{\prime}$ be the point on $C$ that is diametrically opposite to $p_{i}$. The diameter $p_{i} p_{i}^{\prime}$ divides the circle $C$ into two halves. We claim that $p_{i}$ has atmost 1 neighbor in each half. Let, if possible, $p_{i}$ have two neighbors $p_{j}$ and $p_{k}$ in the same half (see Figure 6(i)). We can see that $\angle p_{i} p_{j} p_{i}^{\prime}=90^{\circ}$. Since $p_{i}, p_{j}, p_{k}, p_{i}^{\prime}$ are in convex position, we have $\angle p_{i} p_{j} p_{k}>\angle p_{i} p_{j} p_{i}^{\prime}$. Thus, $\angle p_{i} p_{j} p_{k}>90^{\circ}$ But, since $\left(p_{i}, p_{k}\right)$ is an edge, $\angle p_{i} p_{j} p_{k}<90^{\circ}$ (by Lemma2.1). Hence a contradiction.

Since, each point $p_{i} \in P$ has atmost 2 neighbors (atmost one in each half), the edge complexity of $G_{P}$ is atmost $n$.

This bound is exact, since we can always construct a $G_{P}$ with $n$ edges.

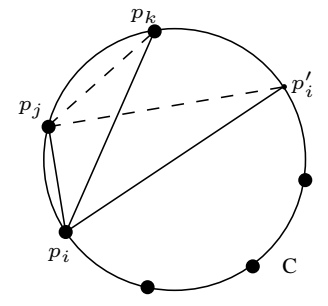

(i)

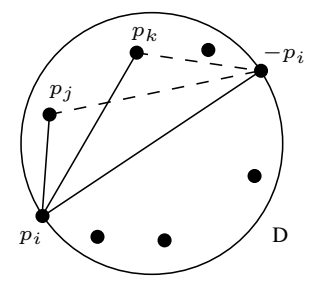

(ii)

Figure 6: (i) Points on a circle $C$ (ii) Centrally symmetric point set with diameter pair $p_{i},-p_{i}$

\subsubsection{Centrally symmetric convex point set}

Next, we consider the case of $P$ being in centrally symmetric convex position. We prove that any locally gabriel graph on $P$ has atmost $2 n-3$ edges. Our proof is an adaptation of [1], where it was proved that the unit distance graph on centrally symmetric convex point sets has atmost $2 n-3$ edges.

Lemma 4.4 Let $P=\left\{p_{1},-p_{1}, p_{2},-p_{2} \ldots, p_{n / 2},-p_{n / 2}\right\}$ be $n$ points in centrally symmetric convex position. Let $G_{P}$ be any locally gabriel graph on $P$. $G_{P}$ has atmost $2 n-3$ edges

Proof. In [1], it is shown that the diameter pair (pair that is furthest apart) in any centrally symmetric convex point set must be of the form $\left(p_{m},-p_{m}\right)$, for some $m$. Let $\left(p_{i},-p_{i}\right)$ be the diameter pair in $P$.

If $\left(p_{i},-p_{i}\right)$ is an edge in $G_{P}$, we can show (by a similar argument as below) that $p_{i},-p_{i}$ has atmost 1 neighbors in $P$. Thus, $G_{P}$ would have atmost $n-1$ edges. Therefore, let us assume that $\left(p_{i},-p_{i}\right)$ is not an edge in $G_{P}$.

Consider the closed disk $D$ with $p_{i}$ and $-p_{i}$ as diameter. Since $P$ is centrally symmetric, all the points in $P$ must lie in $D$. The diameter $p_{i},-p_{i}$ divides the disk $D$ into two halves. We claim that $p_{i}$ has atmost 1 neighbor in each half. Let, if possible, $p_{i}$ have two neighbors $p_{j}$ and $p_{k}$ in the same half (see Figure 6 ii)). Since, $p_{j}$ lies in $D, \angle p_{i} p_{j}-p_{i} \geq 90^{\circ}$. Also, since $p_{i}, p_{j}, p_{k},-p_{i}$ are in convex position, $\angle p_{i} p_{j} p_{k}>$ 
$\angle p_{i} p_{j}-p_{i}$. Thus, $\angle p_{i} p_{j} p_{k}>90^{\circ}$. Since $\left(p_{i}, p_{k}\right)$ is an edge, $\angle p_{i} p_{j} p_{k}<90^{\circ}$ (by Lemma 2.1). Hence a contradiction.

$p_{i}$ has atmost 2 neighbors in $G_{P}$. By the same argument, $-p_{i}$ also has atmost 2 neighbors. Removing $p_{i}$ and $-p_{i}$ from $P$ and recursing on the remaining point set (which is also centrally symmetric), we have $P(n) \leq P(n-2)+4 ; P(2)=1$. This gives $P(n) \leq 2 n-3$.

We can achieve an almost tight lower bound using the following construction:

Let $P$ be a set of $n$ points defined by $P=\{(-1, i) \cup(1, i),-n / 4 \leq i<n / 4\}$. $P$ consists of equally spaced integer gridpoints on the vertical lines $x=-1$ and $x=1$ ( $n / 2$ points in each line). It is easy to see that $P$ is centrally symmetric about the origin. The edges of $G_{P}$ are defined as follows:

(i) Add $n-4$ edges of the form $((-1, i),(-1, i+2))$ and $((1, i),(1, i+2))$ for all $-\frac{n}{4} \leq i<\frac{n}{4}-2$.

(ii) Add $n-4$ edges of the form $((-1, i),(1, i+1))$ and $((-1, i),(1, i-1))$ for all $-\frac{n}{4}-1 \leq i<\frac{n}{4}-1$.

It can be easily verified that these edges satisfy the locally gabriel condition. Thus, the edge complexity of $G_{P}$ is $2 n-8$.

\subsection{Bounds for Convex Point Sets}

In this subsection, we consider an arbitrary convex point set $P$. We prove that the edge complexity of any LGG on $P$ is $O(n \log n)$. The proof is a straightforward extension of the argument given in [5], which proved that the unit distance graph on convex point sets has $O(n \log n)$ edges.

Lemma 4.5 Let $P$ be a set of $n$ points in convex point set and let $G_{P}$ be any locally gabriel graph on $P . G_{P}$ has $O(n \log n)$ edges.

Proof. We use the clever recursive method given in [5]. We will describe the method briefly, for sake of completeness. Refer to [5] for details. We can partition $P$ into $Q$ and $R$ using the topmost and bottommost point of $P$ (antipodal pair). Note that $Q$ is left half convex and $R$ is right half convex. In fact, we can perform a partition using any of the antipodal pairs, such that the two parts are half convex sets (for an appropriate reference axis). The basic idea behind the recursive method in [5] is to use the above fact to divide $P$ using two such partitions such that we have two subproblems of size atmost $3 n / 4$ and the edges at this level of recursion are edges within the four half convex sets. The number of such edges is $O(n)$ using Lemma 4.2. The edge complexity of $G_{P}$ is thus $O(n \log n)$

For convex point sets, the best known lower bound is $2 n-3$. 


\section{Independent Sets}

In this section, we show that any $L G G$ on any $n$ point set contains an independent set of size at least $\Omega(\sqrt{n} \log n)$.

We first show an elementary argument that constructs an independent set of size at least $\frac{\sqrt{n}}{2}$ in a $n$ point set. A set of points ordered by their abscissa is called a monotonic sequence if the ordinates of the points are either monotonically non-increasing or monotonically non-decreasing.

Lemma 5.1 Let $G_{P}$ be any $L G G$ on a monotonic sequence $P$ with $n$ points. $G_{P}$ has an independent set of size at least $\frac{n}{2}$.

Proof. Let us denote the first and the last vertices of the monotonic sequence $P$ as terminal vertices. We show that in any $L G G$ on $P$, a terminal vertex has degree at most one. On the contrary let us assume that a terminal vertex $v$ is incident to vertices $v_{1}$ and $v_{2}$ and the vertices appear in the sequence as $v, v_{1}$ and $v_{2}$. An axis parallel rectangle with $v v_{2}$ as diagonal will contain $v_{1}$ inside or on the boundary of it. It implies that edges $\left(v, v_{2}\right)$ and $\left(v, v_{1}\right)$ conflict with each other. Thus, $v$ has at most one edge incident to it. Now, add the terminal vertex to the independent set and remove it along with its neighbor (if it exists) from the sequence. In each iteration at most two vertices are removed and one vertex is added to the independent set. Thus, the independent set has size at least $\frac{n}{2}$.

Erdos and Szekeres [7] showed that a set of $n$ points will have a monotonic sequence of size at least $\sqrt{n}$. One such sequence can be computed in $O(n \log n)$ time by an algorithm proposed by Hunt and Szymanski [10]. By Lemma 5.1, any induced $L G G$ on this monotonic sequence has an independent set of size at least $\frac{\sqrt{n}}{2}$.

Now, we show that any $L G G$ on any point set with $n$ points contains an independent set of size at least $\Omega(\sqrt{n} \log n)$. In a graph $G=(V, E)$ for any $u \in V$, let us define $N(u)=\{v \mid(u, v) \in E\}$. A graph is said to have sparse neighborhood if for any $u \in V$, the chromatic number of the subgraph induced over vertices $\{u\} \cup N(u)$ is a constant. We show that any $L G G$ with $n$ vertices will have an independent set of size $\Omega(\sqrt{n} \log n)$ by using Theorem 5.1 where the sparse neighborhood property of LGGs (shown in the Lemma 5.2) is applied.

Theorem 5.1 (Alon [2]) Let $G=(V, E)$ be a graph on $n$ vertices with average degree $t \geq 1$ in which for every vertex $v \in V$ the induced subgraph on the set of all neighbors

of $v$ is $r$-colorable. Then, the independence number of $G$ is at least $\frac{c}{\log (r+1)} \frac{n}{t} \log t$, for some absolute positive constant $c$.

Lemma 5.2 Let $G_{P}$ be any $L G G$ on any point set $P$ and $u$ be an arbitrary vertex in $G$. The induced subgraph over the vertices $\{u\} \cup\{N(u)\}$ is 4-colorable.

Proof. Let vertex $u$ be adjacent to $v_{1}, v_{2}, \ldots, v_{k}$. Let us consider the induced subgraph over these vertices. We show that any vertex say $v_{1}$ has at most one incident edge on either side of the line passing through $u$ and $v_{1}$. On the contrary let us assume that there are two vertices $v_{2}$ and $v_{3}$ adjacent to $v_{1}$ on the same side of line $\overline{u v_{1}}$. Let us analyze all the possible cases. 

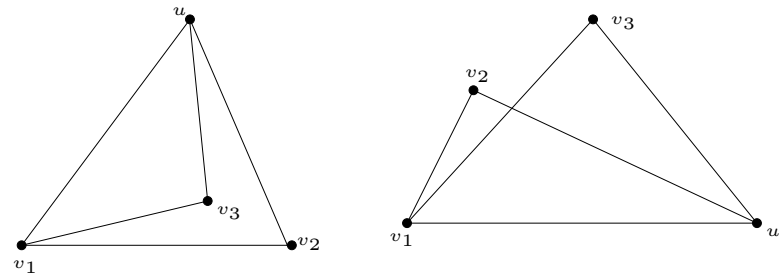

Figure 7: Possible placement of neighborhood in $L G G$

- All the four vertices $\left(u, v_{1}, v_{2}\right.$ and $\left.v_{3}\right)$ cannot be collinear otherwise at least two vertices (say $v_{1}$ and $v_{2}$ w.l.o.g.) lie on the same side of $u$ and the edges $\left(u, v_{1}\right)$ and $\left(u, v_{2}\right)$ would conflict with each other.

- Let us consider the case when three vertices are collinear. It can be trivially verified that $v_{1}, v_{2}$ and $v_{3}$ cannot be collinear due to $L G G$ constraints. Similarly $u, v_{1}$ and $v_{2}$ (or $v_{3}$ ) also cannot be collinear due to $L G G$ constraints. If $v_{2}, v_{3}$ and $u$ are collinear then $u$ must lie in between $v_{2}$ and $v_{3}$. It contradicts with the assumption that $v_{2}$ and $v_{3}$ lie on the same side of $\overline{u v_{1}}$.

- Let us consider the case when convex hull of these four vertices is a triangle and another vertex lies inside this triangle as shown in Figure 7 (a). Since it is assumed that $v_{2}$ and $v_{3}$ lie on the same side of $\overline{u v_{1}}, u$ and $v_{1}$ must be the vertices of this triangle. Let us assume that vertex $v_{3}$ lies inside $\triangle u v_{1} v_{2}$. Since $\left(u, v_{1}\right),\left(u, v_{2}\right)$ and $\left(u, v_{3}\right)$ do not conflict with each other, both $\angle u v_{3} v_{1}$ and $\angle u v_{3} v_{2}$ should be less than $\frac{\pi}{2}$, which is not possible in this configuration.

- The last case is when all the vertices are in convex position and form a quadrilateral. Lets assume w.l.o.g. that $u v_{1} v_{2} v_{3}$ is a convex quadrilateral as shown in Figure 7b). By Lemma 2.1, $\angle u v_{1} v_{2}<\frac{\pi}{2}$ (due to edges $u v_{1}$ and $u v_{2}$ ), $\angle v_{1} v_{2} v_{3}<\frac{\pi}{2}$ (due to edges $v_{1} v_{3}$ and $v_{1} v_{2}$ ), $\angle v_{2} v_{3} u<\frac{\pi}{2}$ (due to edges $u v_{2}$ and $u v_{3}$ ), $\angle v_{3} u v_{1}<\frac{\pi}{2}$ (due to edges $v_{1} u$ and $v_{1} v_{3}$ ) and . But in a quadrilateral at least one of the internal angle should be greater than or equal to $\frac{\pi}{2}$. Hence, it leads to a contradiction.

Hence any vertex $v_{i} \in N(u)$ has at most two neighbors apart from $u$ in the induced subgraph on neighborhood of $u$. Thus, the degree of any vertex $v_{i}$ for $1 \leq i \leq k$ is at most 3 . Therefore, this induced subgraph is 4-colorable.

Theorem 5.2 Let $G_{P}$ be any $L G G$ on a $n$ point set. $G_{P}$ has an independent set of size $\Omega(\sqrt{n} \log n)$.

Proof. Since an $L G G$ has a maximum of $O\left(n^{\frac{3}{2}}\right)$ edges [12], the average degree of a vertex is $O(\sqrt{n})$. Substituting $t=O(\sqrt{n})$ and $r=4$ in Theorem 5.1, the desired bound is obtained. 


\section{Conclusion}

In this paper, we have shown improved bounds on the maximum edge complexity of locally gabriel graphs. There is still a gap between our lower bound of $\Omega\left(n^{5 / 4}\right)$ and the best known upper bound of $O\left(n^{3 / 2}\right)$. It is an interesting problem to narrow this gap. We have shown tight linear bounds for various subclasses of convex pointsets. But, for a general convex point sets, the best lower bound on edge complexity of locally gabriel graphs is $2 n-3$, while the upper bound is $O(n \log n)$. Can one obtain tight bounds? Finally, we have shown that any LGG on any $n$ pointset has an independent set of size $\Omega(\sqrt{n} \log n)$. There is no known non-trivial upper bound. It is an interesting problem to improve upon these bounds.

\section{References}

[1] B. M. Ábrego and S. Fernández-Merchant. The unit distance problem for centrally symmetric convex polygons. Discrete \& Computational Geometry, 28(4):467-473, 2002.

[2] N. Alon. Independence numbers of locally sparse graphs and a ramsey type problem. Random Struct. Algorithms, 9(3):271-278, 1996.

[3] P. Bose, P. Morin, I. Stojmenović, and J. Urrutia. Routing with guaranteed delivery in ad hoc wireless networks. In Proceedings of the 3rd international workshop on Discrete algorithms and methods for mobile computing and communications, pages 48-55, New York, NY, USA, 1999. ACM.

[4] P. Brass, W. Moser, and J. Pach. Research Problems in Discrete Geometry. Springer, 2005.

[5] P. Braß and J. Pach. The maximum number of times the same distance can occur among the vertices of a convex $\mathrm{n}$-gon is o(n $\log \mathrm{n})$. J. Comb. Theory, Ser. A, 94(1):178-179, 2001.

[6] H. Edelsbrunner and P. Hajnal. A lower bound on the number of unit distances between the vertices of a convex polygon. Journal of Combinatorial Theory, Series A, 56(2):312 - 316, 1991.

[7] P. Erdos. On sets of distances of n points. Amercan Math. Monthly, 53:248-250, 1946.

[8] Z. Füredi. The maximum number of unit distances in a convex n-gon. J. Comb. Theory, Ser. A, 55(2):316-320, 1990.

[9] R. K. Gabriel and R. R. Sokal. A new statistical approach to geographic variation analysis. Systematic Zoology, 18(3):259-278, September 1969.

[10] J. W. Hunt and T. G. Szymanski. A fast algorithm for computing longest common subsequences. Commun. ACM, 20:350-353, May 1977.

[11] J. Jaromczyk and G. Toussaint. Relative neighborhood graphs and their relatives. $P$-IEEE, 80:1502-1517, 1992. 
[12] S. Kapoor and X.-Y. Li. Proximity structures for geometric graphs. In International Journal of Computational Geometry and Applications, volume 20, pages 415-429, 2010.

[13] X.-Y. Li, G. Calinescu, and P.-J. Wan. Distributed construction of a planar spanner and routing for ad hoc wireless networks. In in IEEE INFOCOM, pages 1268$1277,2002$.

[14] X.-Y. Li, G. Calinescu, and P.-J. Wan. Distributed construction of planar spanner and routing for ad hoc wireless networks. In INFOCOM, 2002.

[15] R. Pinchasi and S. Smorodinsky. On locally delaunay geometric graphs. In Symposium on Computational Geometry, pages 378-382, 2004.

[16] L. A. Székely. Crossing numbers and hard erdos problems in discrete geometry. Comb. Probab. Comput., 6(3):353-358, Sept. 1997.

[17] E. Szemerdi and W. Trotter. Extremal problems in discrete geometry. Combinatorica, 3:381-392, 1983. 10.1007/BF02579194.

[18] J. Urrutia. Local solutions for global problems in wireless networks. Journal of Discrete Algorithms, 5:395-407, September 2007. 